\title{
MUC1 polymorphism confers increased risk for intestinal metaplasia in a Colombian population with chronic gastritis
}

\author{
Filipe Silva ${ }^{*}{ }^{1}$, Filipa Carvalho ${ }^{1,2}$, António Peixoto ${ }^{1}$, Ana Teixeira ${ }^{1}$, Raquel Almeida ${ }^{1}$, \\ Celso Reis ${ }^{1}$, Luis Eduardo Bravo ${ }^{3}$, Luis Realpe ${ }^{4}$, Pelayo Correa ${ }^{5}$ and Leonor David ${ }^{1,2}$
}

\author{
${ }^{1}$ Institute of Molecular Pathology and Immunology of the University of Porto, IPATIMUP, Porto, Portugal; ${ }^{2}$ Medical \\ Faculty, University of Porto, Porto, Portugal; ${ }^{3}$ Department of Pathology, Universidad del Valle, Cali, Colombia; \\ ${ }^{4}$ Hospital Departamental, Pasto, Colombia; ${ }^{5}$ Department of Pathology, Louisiana State University Health Sciences \\ Center, New Orleans, LA, USA
}

Gastric cancer (GC) stands as the second most common cause of cancer death for males worldwide, and intestinal metaplasia (IM) is a lesion that precedes GC development. In previous works it was shown that polymorphisms of MUC1 gene are associated with increased risk for GC and IM. The aim of the present study was to evaluate MUC1 gene polymorphism in patients with chronic gastritis from Colombia. A Portuguese population of patients with chronic gastritis was used for comparative purposes. A total of 67 Colombian cases and 52 Portuguese cases were analysed by restriction analysis and Southern blotting. MUC1 allele frequencies were significantly different between the two populations, with an overall prevalence of smaller alleles in Colombian samples. Colombian cases showed a lower prevalence of individuals homozygous for small MUC1 mucins in cases without IM (62.5\%) when compared with cases with IM (86.0\%). The same trend, although not statistically significant, is observed in the Portuguese population. In conclusion, our study shows that Colombian patients with chronic gastritis have a significantly higher prevalence of small MUC1 alleles than the Portuguese population. Our study also shows that small MUC1 genotypes are associated with increased risk for IM development in Colombian patients.

European Journal of Human Genetics (2003) 11, 380-384. doi:10.1038/sj.ejhg.5200978

Keywords: gastritis; intestinal metaplasia; mucin; MUC1; polymorphism

\section{Introduction}

Gastric cancer stands as the second most common cause of cancer death for males worldwide. ${ }^{1}$ The gastric carcinogenesis pathway is still poorly understood, but most studies indicate that intestinal metaplasia (IM) is a lesion of the gastric mucosa that precedes gastric carcinoma (GC) development. ${ }^{2}$ Therefore, it is tempting to identify risk

*Correspondence: F Silva, IPATIMUP, Rua Dr Roberto Frias s/n, 4200 Porto, Portugal. Tel: +351 22 5570700; Fax: +351 22 5570799;

E-mail: fsilva@ipatimup.pt

Received 6 August 2002; revised 10 January 2003; accepted 5 February 2003 factors associated with IM as a way of progressing in the understanding of gastric carcinogenesis.

Mucins are heavily glycosylated proteins that constitute the major component of the mucous protective layer above mucosal surfaces. ${ }^{3}$ MUC1 has been described as a membrane-associated molecule expressed on the apical membrane of various glandular epithelial cells and hematopoietic cells. $^{4,5}$ In 1997, our group has shown that individuals with MUC1 gene containing a small number of tandem repeats are at increased risk for GC development. ${ }^{6}$ The rationale underlying that first study was that the genetic variation in size, common to most mucin genes, ${ }^{7}$ might have an influence on the protective capabilities of 
the final mucin product. The MUC1 mucin was chosen as a target since it is expressed in gastric mucosa, and the gene polymorphism was demonstrated to have a direct effect on the size of the protein product. ${ }^{8}$ We have then shown that, in the Portuguese population, the same MUC1 genetic polymorphism is associated with increased risk for development of IM, mostly incomplete IM subtype. ${ }^{9}$

In the present study we explore if the MUC1 susceptibility model for GC and IM is transposable to populations with distinct genetic background, in this case a series of individuals with chronic gastritis from a Colombian area with a high incidence of GC.

\section{Materials and methods Populations}

We evaluated the MUC1 gene polymorphism in blood samples from two populations of patients with chronic gastritis. Colombian patients $(n=67)$ were from Nariño, an Andean region of southern Colombia with an extremely high prevalence of GC. The Colombian patients population (age: $49.6 \pm 8.8$ years; male:female ratio $1.5: 1$ ) is mostly formed by 'Mestizos', a mixture of Amerindian and Spaniard ancestries. Portuguese patients $(n=52)$ (age: $48.3 \pm 8.1$; male:female ratio 4.2:1) were recruited during a screening program for (pre)malignant lesions of the gastric mucosa in a northern region of Portugal with a high incidence of gastric cancer. Portuguese patients were all Caucasians.

\section{MUC1 polymorphism analysis}

DNA was isolated from blood samples $(10 \mathrm{ml})$, using a saltchloroform extraction method, as previously described by Mullenbach et al. ${ }^{10}$ DNA samples were digested with AluI (restriction sequence $A G \downarrow C T$ ), which recognizes restriction sites in the regions flanking the tandem repeats of the MUC1 gene. Electrophoretic separation and Southern blotting were performed using the conditions previously described. ${ }^{6}$

All the alleles identified were ranked and numbered from 1 to 20 according to their size and by comparison with a ladder of alleles previously characterized. ${ }^{11}$ Allele 1 represents the largest allele and allele 20 represents the smallest allele. The sizes of the different MUC1 alleles were obtained by comparison with the mobility of the marker $\lambda$ HindIII (Amersham).

Allele frequencies and Hardy-Weinberg equilibrium were assayed by GENEPOP software. ${ }^{12}$ The high polymorphism of the MUC1 locus prompted us to group the alleles in large (L) and small (S) categories according to Carvalho et al. ${ }^{6}$ Alleles 1-9 were grouped in the category of large alleles (L) and alleles 10-20 were grouped in the category of small (S) alleles. The partition of the alleles into large and small categories was performed using the median value of the distribution of the alleles in a control population of blood donors. Genotypes were thereafter recoded as LL (MUC1 large VNTR homozygotes), SS (MUC1 small VNTR homozygotes), and LS (heterozygotes for large and small alleles).

\section{Endoscopy and histological characterization of the gastric lesions}

From each patient biopsy specimens were taken from corpus $(n=1)$ and antrum $(n=2)$. Endoscopes were reprocessed after each procedure with adequate clearing and disinfection in a $2 \%$ glutaraldehyde solution according to the ESGE guidelines. ${ }^{13}$

Gastric biopsies for histology studies were fixed in $10 \%$ formalin and routinely embedded in paraffin wax. The histological classification of the biopsies was performed according to the revised version of the Sydney system ${ }^{14}$ into four distinct diagnostic classes: (a) chronic gastritis without atrophy, (b) chronic gastritis with atrophy, (c) chronic gastritis with atrophy and IM, and (d) chronic gastritis with atrophy, IM and dysplasia (Figure 1).

\section{Statistical analysis}

The frequencies of MUC1 alleles in the Colombian population were compared with the frequencies in the Portuguese population. Heterogeneity analysis between populations was based on allelic contingency tables using the STRUC program from the software package GENEPOP.
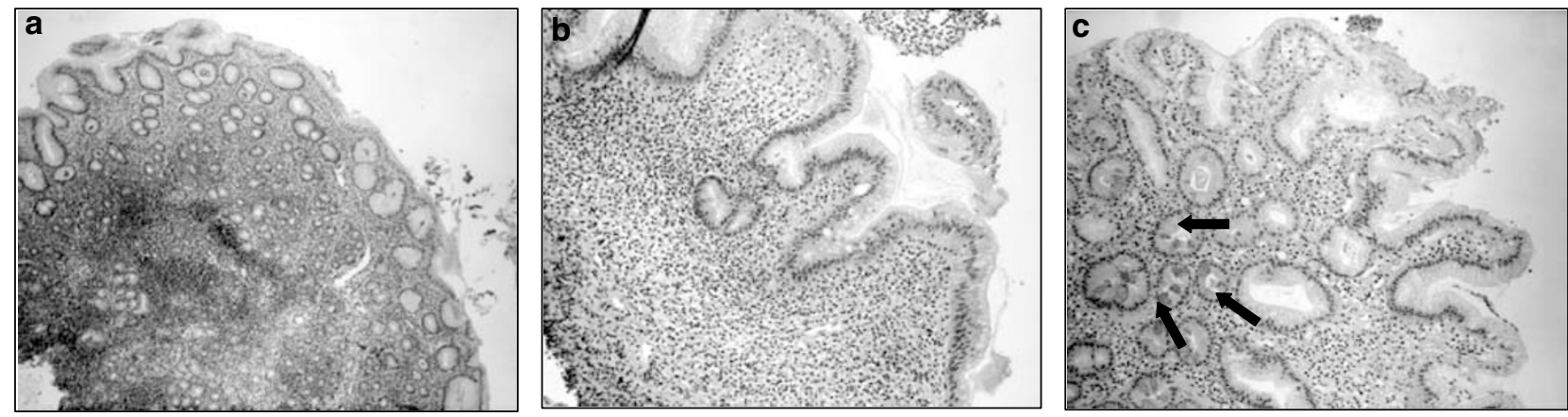

Figure 1 Histology of different diagnostic classes: (a) chronic gastritis without atrophy; (b) chronic gastritis with atrophy; (c) chronic gastritis with atrophy and IM (arrows). 


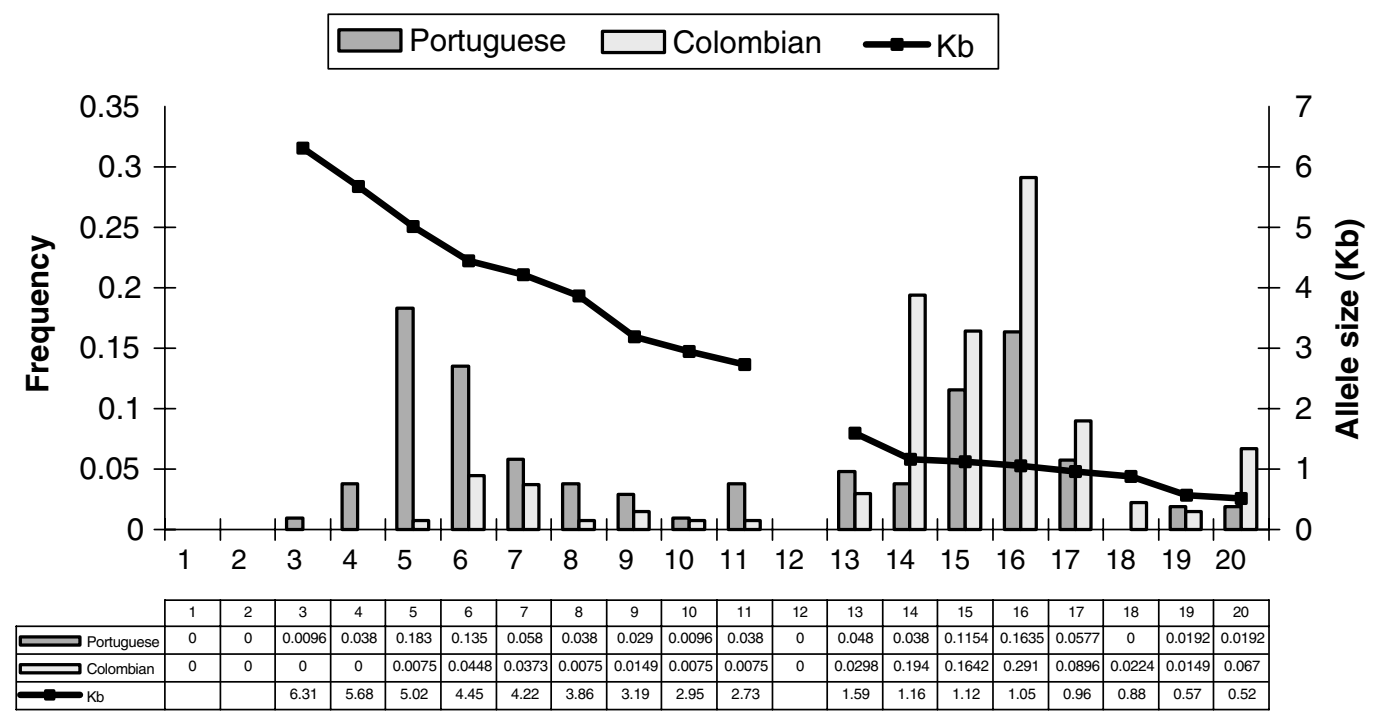

Alleles

Figure 2 Distribution of allele frequencies of MUC1 gene in Colombian $(n=67)$ and Portuguese $(n=52)$ patients with chronic gastritis. Alleles are numbered according to their size by decreasing order of molecular weight (allele 1 is the largest). Molecular weight of the different alleles is also plotted in the figure using the secondary $Y$-axis.

Associations between polymorphisms and the presence of atrophy and IM were evaluated using STATVIEW 4.02 software (SAS Institute, Cary, NC, USA). The Monte Carlo test was also applied (10000 iterations) whenever expected values were less than 5 as described by Sham and Curtis ${ }^{15}$ using the computer program CLUMP. Odds ratio and $95 \%$ confidence limits were determined using the BMDP $4 \mathrm{~F}$ computer program (Statistical Package program BMDP; Los Angeles, CA, USA).

\section{Results}

The analysis of the MUC1 polymorphism in the 67 Colombian patients disclosed 15 alleles combined in 31 distinct genotypes with $85 \%$ of heterozygotes. All the alleles present in the Colombian patients had been previously described in other populations. ${ }^{11}$ Data from Colombian patients were compared with those from a subsample of 52 Portuguese patients with chronic gastritis previously described. ${ }^{9}$ The overall MUC1 allele frequencies distribution of the Colombian population is significantly different from the Portuguese population distribution $(P<0.001)$ (Figure 2$)$. The differences are mainly due to alleles 14 and 16 (small VNTRs), which are significantly over-represented in Colombian cases $(P<0.001$ and $P=0.022$, respectively), while alleles 5 and 6 (large VNTRs) are more prevalent in Portuguese cases $(P<0.001$ and $P=0.019$, respectively). The genotypic distributions are in Hardy-Weinberg equilibrium for both populations.

The gastric lesions observed in Colombian and Portuguese patients were classified in distinct categories
Table 1 Frequency of diagnostic categories in 67 patients from Colombia and 52 patients from Portugal with chronic gastritis

\begin{tabular}{lcr}
\hline Diagnostic categories & $\begin{array}{c}\text { Colombia } \\
\text { Number of } \\
\text { patients (\%) }\end{array}$ & $\begin{array}{c}\text { Portugal } \\
\text { Number of } \\
\text { patients (\%) }\end{array}$ \\
\hline Without atrophy & $11(16.4)$ & $6(11.5)$ \\
With atrophy & $13(19.4)$ & $11(21.2)$ \\
Atrophy with IM & $35(52.3)$ & $35(67.3)$ \\
Atrophy with IM and dysplasia & $8(11.9)$ & $0(0.0)$ \\
\hline
\end{tabular}

(Table 1). The Colombian population showed atrophy in 56 cases $(83.6 \%)$, and IM in 43 cases $(64.2 \%)$, eight of them with dysplasia. The Portuguese population showed atrophy in 46 cases $(88.5 \%)$, and intestinal IM in 35 cases $(67.3 \%)$; no dysplastic lesions were observed in this population.

The frequency of MUC1 recoded genotypes showed significant differences according to the presence or absence of IM in the Colombian population (Table 2). Colombian cases showed a lower prevalence of SS genotypes in cases without IM (62.5\%) when compared with cases with IM $(86.0 \%)$. The same trend, though not statistically significant as previously published for the whole population, ${ }^{9}$ is observed in the Portuguese subsample (Table 2). No associations were found for classes with atrophy or dysplasia (data not shown).

The odds ratio for the development of IM was calculated according to the MUC1 genotypes - the cases homozygous 
for large alleles (LL) were deleted from the analysis since only one case with an LL genotype was observed in the Colombian population. The presence of small VNTRs in homozygosity confers an increased risk for IM development (odds ratio 3.7) while the heterozygotes are 'protected' from the development of IM (odds ratio 0.2). The odds ratio for development of atrophy or dysplasia is not statistically significant (data not shown). A trend for an increased risk of developing IM in individuals with small VNTRs homozygosity was also observed in the Portuguese population (Table 3 ).

\section{Discussion}

In the present study we evaluated a cohort of Colombian patients with chronic gastritis from a region (Nariño) with a high incidence of gastric cancer. ${ }^{16}$ We have shown that MUC1 allele frequencies of the Colombian patients are significantly different from the allele frequencies of a subsample of a previously described Portuguese population of patients with chronic gastritis. ${ }^{9}$ Individual analysis of the alleles shows that Colombian patients have a significantly higher frequency of small VNTR alleles (alleles 14 and 16), while large VNTR alleles (alleles 5 and 6) have a significantly higher frequency in the Portuguese patients. The data show that Colombian and Portuguese patients' populations with chronic gastritis are genetically different for the MUC1 polymorphism, probably reflecting population ancestry and locus evolution.

Both the Colombian and the Portuguese patients with chronic gastritis have a high prevalence of IM (64.2 and $67.3 \%$, respectively). The high frequency of IM in the two

Table 2 Frequency of chronic gastritis with and without IM according to MUC1 recoded genotypes in the Colombian and Portuguese populations

\begin{tabular}{lcccr}
\hline & \multicolumn{3}{c}{ Colombia } & \multicolumn{2}{c}{ Portugal } \\
MUC1 recoded & Without IM & With IM & Without IM & With IM \\
genotypes & N (\%) & N (\%) & N (\%) & N (\%) \\
\hline LL & $0(0.0)$ & $1(2.3)$ & $5(29.4)$ & $8(22.9)$ \\
LS & $9(37.5)$ & $5(11.6)$ & $8(47.1)$ & $17(48.6)$ \\
SS & $15(62.5)$ & $37(86.0)$ & $4(23.5)$ & $10(28.6)$ \\
& $P=0.037$ & & $P=0.856$ \\
\hline
\end{tabular}

populations fits with the presence of a high incidence of GC in both settings. ${ }^{17,18}$ The association between SS MUC1 genotypes and IM that we observed in the Colombian population (odds ratio 3.7) fits with previous observations in a Portuguese population. ${ }^{9}$ Both observations suggest that in lesions of chronic gastritis, metaplastic transformation is associated with SS MUC1 genotypes. Similarly, a protective role for IM development is associated with MUC1 heterozygosity (LS) in the Colombian patients, as previously observed in the Portuguese population. ${ }^{9}$

The observations reported in the present study, together with previous publications, ${ }^{6,9}$ reinforce that small MUC1 VNTRs homozygosity is associated with increased risk for the development of $\mathrm{IM}^{9}$ and gastric carcinoma. ${ }^{6}$ However, recent data from the literature show that allele size does not influence the levels of MUC1 expression in the gastric mucosa. ${ }^{19}$ The putative biological significance of MUC1 VNTR variability remains elusive and should be addressed in future biochemical studies aiming to explore the effect of mucin size (VNTR alleles) in the adhesion of pathogens and mucin oligomerization.

In conclusion, our study shows that Colombian and Portuguese patients with chronic gastritis are genetically different for the MUC1 VNTR locus. Our study also shows that MUC1 small VNTRs genotypes confer increased susceptibility for IM development in the context of chronic gastritis in Colombian patients. The results support the involvement of MUC1 VNTR in the susceptibility for development of IM and GC in populations with different genetic background.

\section{Acknowledgements \\ We thank Prof. Manuel Sobrinho-Simões for many helpful suggestions and a critical reading of the manuscript. We are grateful to the following departments where patients' data and samples were collected: Estaleiros Navais de Viana do Castelo, Departments of Surgery 3 and Gastroenterology of Hospital S. João, Porto, Portugal. This work was supported by: Administração Regional de Saúde do Norte; Fundação para a Ciência e Tecnologia e Programa Operacional Ciência, Tecnologia, Inovação do Quadro Comunitário de Apoio III (Grant: SFRH/BPD/3622/2000; Projects SAU/14111/1998 and POC- TI/CBO/39075/2001); Fundação Luso Americana para o Desenvolvi- mento (Project 173/2002); Fundação Calouste Gulbenkian (FC-54918) and PO1 CA 28842 of the National Cancer Institute, NIH, USA.}

Table 3 Odds ratio (95\% confidence limits) for the development of IM in patients with chronic gastritis from Colombia and Portugal, according to MUC1 polymorphism

\begin{tabular}{lcccc}
\hline $\begin{array}{l}\text { MUC1 recoded genotypes } \\
\text { with small VNTRs }\end{array}$ & Without IM (95\% cl) & With IM (95\% cl) & Without IM (95\% cl) & Wortugal \\
& & & & \\
Homozygous (SS) & $0.2(0.1-0.9)^{*}$ & $3.7(1.1-12.2)^{*}$ & $0.7(0.2-2.9)$ & $2.2(0.5-8.6)$ \\
Heterozygous (LS) & $4.6(1.3-15.9)^{*}$ & $0.2(0.1-0.8)^{*}$ & $0.9(0.3-3.0)$ & $1.1(0.3-3.4)$ \\
\hline
\end{tabular}

*Significant OR. 


\section{References}

1 Ferlay J, Bray F, Pisani P, Parkin DM: GLOBOCAN 2000: cancer incidence, mortality and prevalence worldwide, version 1.0. IARC Cancer Base no. 5. Lyon: IARC Press, 2001.

2 Correa P: Human gastric carcinogenesis: a multistep and multifactorial process - First American cancer society award lecture on cancer epidemiology and prevention. Cancer Res 1992; 52: 6735-6740.

3 Gendler SJ: MUC1 the renaissance molecule. J Mammary Gland Biol Neoplasia 2001; 6: 339-353.

4 Gendler SJ, Spicer AP: Epithelial mucin genes. Annu Rev Physiol 1995; 57: 607-634.

5 Dent GA, Civalier CJ, Brecher ME, Bentley SA: MUC1 expression in hematopoietic tissues. Am J Clin Pathol 1999; 111: 741-747.

6 Carvalho F, Seruca R, David L et al: MUC1 gene polymorphism and gastric cancer - an epidemiological study. Glycoconj J 1997; 14: 107-111.

7 Fowler J, Vinall L, Swallow D: Polymorphism of the human MUC genes. Front Biosci 2001; 6: 1207-1215.

8 Swallow D, Gendler S, Griffiths B, Corney G, TaylorPapadimitriou J, Bramwell ME: The human tumour-associated epithelial mucins are coded by an expressed hypervariable gene locus PUM. Nature 1987; 328: 82-84.

9 Silva F, Carvalho F, Peixoto A et al: MUC1 gene polymorphism in the gastric carcinogenesis pathway. Eur J Hum Genet 2001; 9: 548-552.

10 Mullenbach R, Lagoda PJ, Welter C: An efficient salt-chloroform extraction of DNA from blood and tissues. Trends Genet 1989; 5: 391.

11 Carvalho F, Peixoto A, Steffensen R, Amorim A, David L, Sobrinho-Simões M: MUC1 gene polymorphism does not explain the different incidence of gastric cancer in Portugal and Denmark. Ann Hum Genet 1999; 63: 187-191.

12 Raymond M, Rousset F: GENEPOP (version 1.2): a population genetics software for exact tests and ecumenicism. I Heredity 1995; 86: 248-249.

13 The European Society of Gastrointestinal Endoscopy: Guidelines on cleaning and desinfection in GI endoscopy. Update 1999. Endoscopy 2000; 32: 77-80.

14 Dixon MF, Genta RM, Yardley JH, Correa P: Classification and grading of gastritis. The updated Sydney system. Am J Surg Pathol 1996; 20: 1161-1181.

15 Sham PC, Curtis D: Monte Carlo tests for associations between disease and alleles at highly polymorphic loci. Ann Hum Genet 1995; 59: 97-105.

16 Correa P, Fontham ET, Bravo JC et al: Chemoprevention of gastric dysplasia: randomized trial of antioxidant supplements and anti-helicobacter pylori therapy. I Natl Cancer Inst 2000; 92: 1881-1888.

17 Filipe MI, Jass JR: Intestinal metaplasia subtypes and cancer risk; in Filipe MI, Jass JR (eds.): Gastric carcinoma. Edinburgh: Churchill Livingstone, 1986, pp 87-115.

18 Rokkas T, Filipe MI, Sladen GE: Detection of an increased incidence of early gastric cancer in patients with intestinal metaplasia type III who are closely followed up. Gut 1991; 32: 1110-1113.

19 Vinall LE, King M, Novelli $M$ et al: Altered expression and allelic association of the hypervariable membrane mucin MUC1 in Helicobacter pylori gastritis. Gastroenterology 2002; 123: 41-49. 